\title{
Chapter 9 \\ Paths to the Way We Live, Teach and Learn
}

In addition to the scientific applications, are there any personal benefits for the reader? Are there any messages which can be kept in mind that would affect our everyday lives? In order to answer this, consider Fig. 9.1. This figure represents an abstract map of a city showing its core at the top and boundaries at the bottom and also some strategic public transportation points where one can switch between transportation devices (buses, trams, underground, etc.). Now let's assume that we are newcomers to the city without any knowledge about the possible transportation options. Our first trip on our first day in the city is to travel from our Hotel $(\mathrm{H})$ to the Bank (B). Now let's assume that we have a "path oracle" (e.g., tourist information) which can be asked for paths between arbitrary points in the city. This oracle always recommends the shortest path between the source and destination. For the trip between the Hotel and the Bank, the oracle gives us the green path, which requires three changes at intermediate transfer points and is the shortest in terms of distance. So, from now on, we can reach the Bank from the Hotel and withdraw money. On the next morning, we want to see the Castle (C), for which the oracle gives us the olive colored path. This has similar characteristics to the green path: three changes and a small traveling distance. On the third day, we plan to withdraw some cash at the Bank and than head straight to the Castle, but when we ask the tourist information, we realize that they are closed. So we are left on our own. What can we do? Well, we know the shortest path from the Bank to the Hotel, and another shortest path from the Hotel to the Castle, so we can travel from the Bank to the Hotel on the green path, then from the Hotel to the Castle on the olive path. This journey will take seven changes at various transfer points.

Now let's turn back the hands of time and assume that we are again newcomers and do the same thing but with a slightly different oracle. This second, more professional, tourist center gives us short paths that preferably follow the hierarchy of the city, i.e., first heading towards its center (upwards), then heads sideways to the border of the city. For our first day trip between our Hotel and the Bank, this second oracle reveals the magenta path trough the Town Hall (T). This trip requires three changes, but slightly longer traveling distance compared to the shortest path 


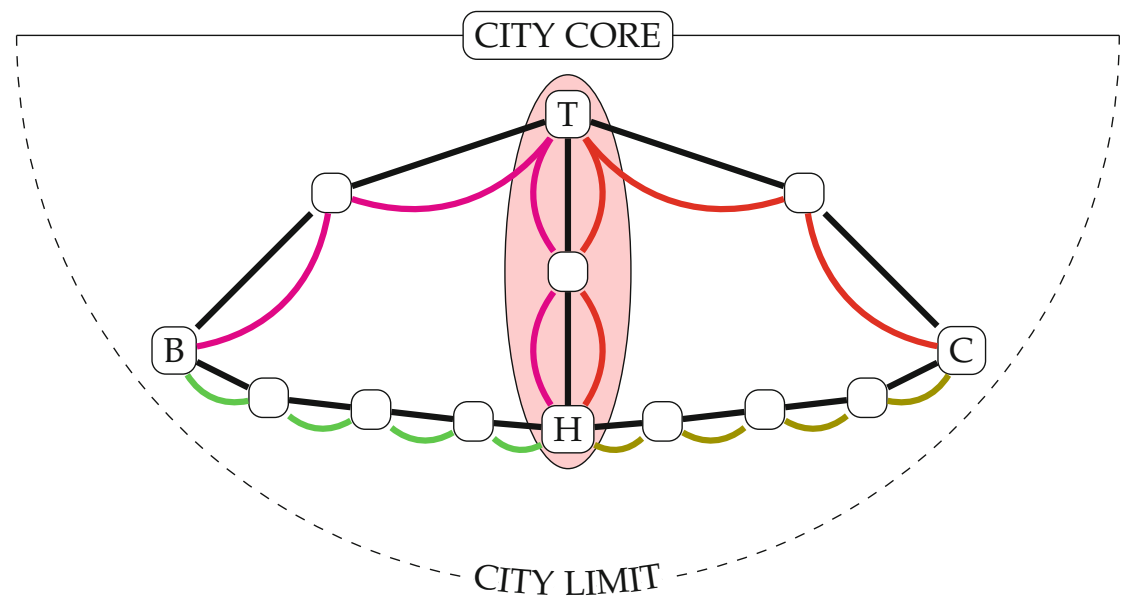

Fig. 9.1 Role of shortest and regular paths

as this goes into the city first and then out to the destination. On the second day, the oracle tells us the red path for our trip between the Hotel and the Castle, which has pretty similar characteristics to the magenta path, also passing by the Town Hall. In the morning of the third day, we need to go from the Bank to the Castle but the employees of the tourist center went on a team-building activity. Again, we are alone in finding a way around! Now if we combine our two paths from the Bank to the Hotel and from the Hotel to the Castle we obtain the following route plan: Bank $\rightarrow \ldots \rightarrow$ Town Hall $\rightarrow \ldots \rightarrow$ Hotel $\rightarrow \ldots \rightarrow$ Town Hall $\rightarrow \ldots \rightarrow$ Castle. But wait a minute, we made an unnecessary by-pass between the Town Hall and the Hotel. We can completely skip this loop and finally take our trip by using the Bank $\rightarrow \ldots \rightarrow$ Town Hall $\rightarrow \ldots \rightarrow$ Castle. This path requires three changes and only slightly longer traveling than the shortest path. So, relying on the advices of the second more professional tourist center in the first 2 days, we can come up with a pretty nice and short trip on the third day all by ourselves.

How can it be? Well, we can see that in the second case, when the oracle gives us paths coinciding with the underlying logic of the city, we can learn much more about the city compared to the case when we stick strictly to the shortest paths. The overlap of the two paths ${ }^{1}$ gives the possibility to relate the paths to each other and from these relations, we extract greater knowledge. After a few initial trips following the logic of the city, tourists can usually leave their city maps in the hotel because they can travel between almost any sources and destinations on the map. Now, imagine how you guide somebody who asks for a path towards some place in your city. Do you offer them the shortest path? Well, maybe you don't know the shortest path to the destination after years of living there. But most probably you will offer a path

\footnotetext{
${ }^{1}$ Marked with a pink ellipse in Fig. 9.1.
} 
coinciding with the logic of the city, i.e., transfer somewhere downtown. Isn't it amazing that nature seems to implement exactly this in many real-life systems? The Internet, the air transportation network, even the human brain seems to implement paths like these. Nature is wise enough not to use shortest paths to ensure knowledge accumulation and navigation inside the system. Long paths cannot be used as they are not effective. So, nature implements a trade-off. By using 10-30\% longer paths, it ensures integrity, learnability, and navigation.

Now let's put this imaginative story into a broader context. Consider that we would like to quickly train somebody to travel inside the city by using the public transportation services. In fact, the knowledge we would like to give her is a usable network of transferring points and their relations to arbitrary points in the city. We can take her with us on some trips within the city. What trips should we choose? Well, we would be surprised if somebody would use shortest path trips for this purpose. What is more probable is that we choose trips according to the logic of the city and in a fashion that the back-to-back journeys have common points preferably in the downtown area. These paths obey the hierarchy or the internal logic of the city and will maximize the extractable knowledge over the shortest period of time.

Now suppose that we want to teach something arbitrary (e.g., biology, maths or farming) by giving lectures or presentations. We argue that the knowledge we want to share is not fundamentally different from the knowledge of being a good user of public transportation. In fact, there are studies showing that children with stronger navigation skills among the world's physical objects can perform better in various learning tasks $[19,23,26]$. Although instead of the physical nodes and physical connections (changing stations and various connecting lines), our nodes and links will likely be "virtual", for example notions and relations between them. The knowledge that we would like the audience to have is a network of concepts related to the topic; we would like them to know the main notions of the field and their connections to each other so they can navigate easily between them and come up with their own ideas as soon as possible. What kind of sentences should we choose? How should we structure our teaching materials?

Emma Ledden, author of the \#1 selling "The Presentation Book"[16] says that: "To avoid your presentation becoming muddled, structure it around your core message or messages. These should be illustrated in different ways, revisited and emphasized, so they are understood and remembered." So, during your talks or lectures, you should frequently revisit the core messages and illustrate them in different ways. This sounds quite the same as showing our imaginary friend a variety of trips in the city, with common points in the city center, isn't it? So nice presentations introduce new elements by carefully relating them to the central notions the presentation is built around and thus revisiting the core concepts many times. These "mental" paths over virtual nodes are the exact equivalent of our city trips in the physical world joining the source and destination nodes through strategic intermediate nodes in the city center.

Now we can turn back for a moment to our question raised in the introduction about mind map based presentation tools: what allowed these tools to compete with giants of the IT industry in the area of presentation software? Well, these "revisiting" 
mental paths are nicely visualized by the mind map based tools when zooming from one view to another, which amplifies the memorization of these paths. Showing the context graphically, the audience is visually taken on a path from notion to notion through the whole logical structure of the presentation, similarly to the trips in our imaginary city. We argue that this path visualization feature of mind map based presentation tools can account for their increasing popularity among presenters.

How can we learn something effectively? If we can use paths to increase the efficiency of teaching, it sounds pretty reasonable that we can use them to increase the efficiency of learning too. How can we use paths to learn something? Well, if we accept the idea that the lecturer is trying to communicate a network, through some specific paths they picked, we can make our learning more efficient by enhancing the process of building this network in our mind. For example, when listening, we can continuously try to relate the things we hear to each other. Moreover, we can create quick experiments to see if we can relate some randomly picked concepts on our own. These experiments can enable us to learn how to navigate among the concepts of the field and discover new connections independently. The formulation of good questions can also be aided by such experiments. In our understanding, learning (not lexical, but practical) is pretty much the same as navigation. Do you want to be better at learning? Our findings about paths say: turn off your GPS whenever you can! Forget about always using the shortest paths to reach your goals! You want your children to learn better at school? Our observations say: Let them tramp freely after school (preferably in a safe environment) and self-develop their navigation skills.

There is a famous Hungarian saying: Don't leave the betrodden way for the untrodden one! It is the subject of continuous misinterpretation in Hungary. People usually think that this saying suggests that you should always be on the safe side and you shouldn't try out anything new. This is usually considered as the curse of Hungarians, according to many thinkers, as it seems to provide the recipe for an unsuccessful life. Delving deeper into the real wisdom this saying may offer us something about learning things more efficiently in the long term. Obviously, when you are in a hurry, you attempt to use the shortest path from your current position. Such a path, however, is likely to be an untrodden one. Think about, for example, finding your way through a big city park from its entrance towards one of its exits. It is not likely that a paved road connects the two in a straight line: you have to tread on the grass. The more you walk on the grass, the higher the possibility that you miss a few signposts that stand only by the paved roads. On one hand, you may miss your direction, on the other, even if you find your way, you would not learn anything about the whereabouts of other exits. Surely if you cross the park only once, late for your own wedding, you would not really care. However, if you do that on a daily basis, you will hardly ever learn what the park really looks like. By skipping the overlaps between your paths, you will never see the relation between your paths and you will hardly learn to navigate yourself confidently. If you pick the betrodden instead, i.e., the sometimes curvy, paved roads in the park, you increase the possibility of overlapping and the chance to learn to orient yourself. Preferring the betrodden doesn't mean by default that your paths should not contain untrodden parts, which is quite inevitable in life. You continuously discover new places and 
people, thus it is highly likely that you will have to use at least partially unknown paths to reach them. But the wisdom of this saying is to keep to the betrodden path while it is possible, to relate and integrate the untrodden segments properly, and to finally understand what the big picture really looks like.

Open Access This chapter is licensed under the terms of the Creative Commons Attribution 4.0 International License (http://creativecommons.org/licenses/by/4.0/), which permits use, sharing, adaptation, distribution and reproduction in any medium or format, as long as you give appropriate credit to the original author(s) and the source, provide a link to the Creative Commons licence and indicate if changes were made.

The images or other third party material in this chapter are included in the chapter's Creative Commons licence, unless indicated otherwise in a credit line to the material. If material is not included in the chapter's Creative Commons licence and your intended use is not permitted by statutory regulation or exceeds the permitted use, you will need to obtain permission directly from the copyright holder. 